\title{
Recurrence Spared Parotid Gland in Patient Receiving Definitive Concurrent Chemo-Radiotherapy for Nasopharyngeal Cancer: A Case Report
}

\author{
Hassani Wissal*, Ait Erraisse Mohammed, Farhane Fatim Zahra, Alami Zenab and Bouhafa Touria \\ Radiotherapy department, HASSAN II Hospital, Morroco
}

\begin{abstract}
Nasopharyngeal carcinoma (NPC) is an uncommon cancer in the most. The standard treatment consists of the combination of radiotherapy in IMRT technique and concurrent chemotherapy. Parotid recurrence is an uncommon pattern of locoregional failure after parotid-gland-sparing radiotherapy for NPC. Because its rarity and the low number of clinical cases reported, the management of parotid recurrence is not yet codified. We are reporting a very case treated in our hospital.
\end{abstract}

\section{INTRODUCTION}

Nasopharyngeal carcinoma (NPC) is an uncommon cancer in the most but very common in Morocco. It is a radiosensitive and radio curable cancer, thus, Chemoradiotherapy is the mainstay of NPC treatment [1]. However, radiotherapy for (NPC) using standard techniques is associated with several side effects, such us xerostomia, which are usually permanent and relevant for the quality of life. The development and diffusion of intensity modulated radiation therapy (IMRT) has been a major breakthrough in the treatment of nasopharyngeal carcinoma (NPC); [2,3] allowed a dose escalation to sites of gross disease while reducing the dose given to critical tissues. However, decreasing the dose of radiation to the parotid glands might result in inadequate radiation exposure to microscopic disease in the region of a spared parotid gland. Nevertheless, periparotid recurrence is an uncommon pattern of failure after definitive IMRT for NPC. We report one case of nodal recurrence within a spared parotid gland after IMRT and revie the literature to find other reports, guidelines and recommendations.

\section{CASE PRESENTATION}

A 28-year-old male came to our consultation with a growing cervical adenopathy, a monolatéral fullness and conductive hearing loss. During physical examination and flexible endoscopy, a reddish exophytic mass implanted on the lateral wall of the nasopharynx was located.

A magnetic resonance imaging (MRI) of the face and skull showed an asymmetric mass in the left later wall of the nasopharynx extending to lateral pharyngeal recess, Eustachian tube, with no intracranial extension and bilateral regional adenopathy (level II and III). There were no distant metastases on the body scan. Cervicotomy confirmed the diagnosis of an undifferentiated nasopharyngeal carcinoma.

The multidisciplinary tumor board of our institution, basing upon the endoscopic, clinical and radiological findings, staged the disease as CT2N3M0 and recommended an induction chemotherapy followed by radio-chemotherapy with a curative intent. The patient received induction chemotherapy with 3cycles of CDDP $75 \mathrm{mg} / \mathrm{m}^{2}$ Epiribicine $60 \mathrm{mg} / \mathrm{m}^{2}$ every three weeks. After 3 weeks from the end of chemotherapy, the patient was treated with concurrent radiochemotherapy. Radiotherapy was delivered into IMRT with inverse planning technique (Figure 1). Total dose to high-risk volume was 70 gy in 35 fractions, 2 Gy per fraction. The average bilateral parotid dose was 20Gy. He received concurrent
Quick Response Code:

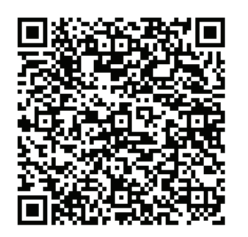

Address for correspondence: Wissal hassani, Radiotherapy department, HASSAN II Hospital, Fes, Morroco

Received: July 08, $2021 \quad$ Published: July 14, 2021

How to cite this article: Hassani W, Ait Erraisse M, Farhane Fatim Z, Alami Z, Bouhafa T. Recurrence spared parotid Gland in Patient Receiving Definitive Concurrent ChemoRadiotherapy for Nasopharyngeal Cancer: A Case Report. 2021- 3(4) OAJBS.ID.000303. DOI: 10.38125/OAJBS.000303 
chemotherapy with cisplatin at the dose of $100 \mathrm{mg} / \mathrm{mg}$ every three weeks for a total of three cycles.

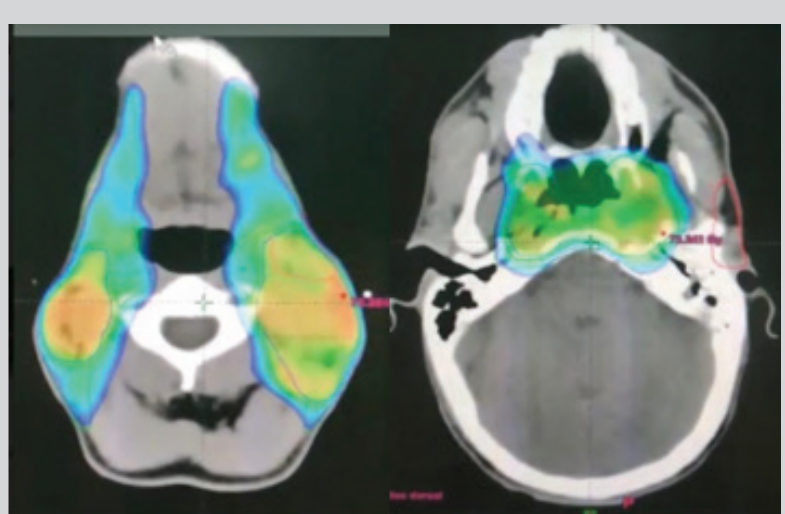

Figure 1: Dose distribution.

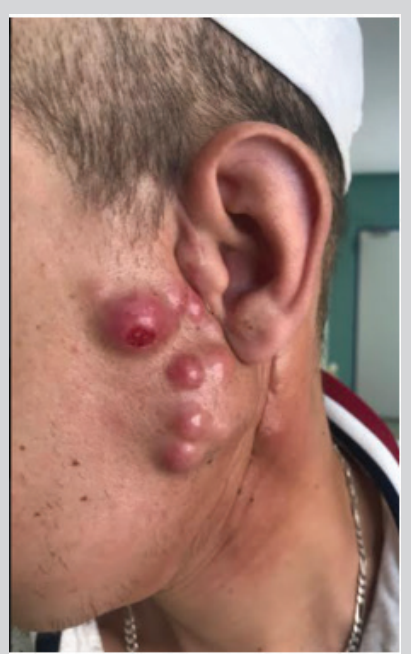

Figure 2: Clinical aspect of UCNT recurrence in left parotid gland.

13 months after the end of radiotherapy, the patient presented a growth regarding left parotid gland (Figure 2). A total parotidectomy with conservation of the nerve trunk of the facial nerve was performed finding on pathological examination a secondary localization of an UCNT. MRI and endoscopy did not find any local recurrence into the nasopharynx.

Since radiotherapy could not be performed due to constraints dose, the multidisciplinary consultation meeting opted for adjuvant chemotherapy docetaxel. The patient died after the second cycle.

\section{DISCUSSION}

In patients who underwent parotid-sparing definitive radiotherapy, the majority of local-regional recurrences are in-field, which represent areas of previous disease judged to be at high risk at the time of radiotherapy planning.[4] Cannon and Lee reported the first recurrence of NPC in a spared parotid gland after definitive parotid gland-sparing IMRT. They suggested that subclinical disease should be suspected inside seemingly harmless nodules for multilevel nodal disease and periparotid nodules at pretreatment imaging [5].

Lin et al reported in 2011 the observation of 3 patients with NPC who developed periparotid failure after definitive parotid glandsparing radiotherapy. They suggested that a later stage of disease, pre-existing metastasis at the periparotid area, and overprotection of the parotid gland during radiotherapy might be related to the development of recurrence in the parotid region. However, any conclusion about the exact reasons of recurrence could be made. These investigators chose parotidectomy followed by adjuvant radiotherapy or chemotherapy, or both, as a salvage procedure. They concluded that early diagnosis and aggressive treatment of periparotid recurrence may help to optimize outcomes [6].

The second study that was interested in the subject was conducted by Cao et al and concerned 10 patients. The proposed treatment consisted of surgery alone for 4 patients, radiotherapy alone for 1 patient while 2 patients received chemotherapy alone, 2 patients received surgery plus chemotherapy, and 1 patient received surgery plus radiotherapy. The authors could not conclude for the optima treatment, but they suggested that salvage treatment can improve survival in this population.

As reported in our patient and in these similar cases, the sparing of the parotid gland can be questioned when lymphonodal metastases are close to the parotid boundaries. Thus, it should be emphasized that the avoidance of xerostomia by IMRT cannot justify any decrease in loco-regional control. Thought optimal treatment is not yet codified, salvage treatment seems to be efficient.

\section{CONCLUSION}

Periparotid recurrence is an uncommon pattern of locoregional failure after parotid-gland-sparing radiotherapy for patients with NPC. Although the etiology of periparotid recurrence is unclear, early diagnosis and salvage treatment may improve outcomes.

\section{REFERENCES}

1. Marnouche (2017) Diagnostic, therapeutic and evolutionary character.

2. Cheng JC, Chao KS, Low D (2001) Comparison of intensity modulated radiation therapy (IMRT) treatment techniques for nasopharyngeal carcinoma. Int J Cancer 96(2): 126-131.

3. Xia P, Fu KK, Wong GW, Akazawa C, Verhey LJ (2000) Comparison of treatment plans involving intensity-modulated radiotherapy for nasopharyngeal carcinoma. Int J Radiat Oncol Biol Phys 48(2): 329-337.

4. Nancy L, Ping X, Nancy JF, Pam A, Clayton A, Jeanne MQ (2003) Intensitymodulated radiation therapy for head-and-neck cancer: the UCSF experience focusing on target volume delineation. International Journal of Radiation Oncology, biology, physics 57(1).

5. Cannon DM, Lee NY (2008) Recurrence in region of spared parotid gland after definitive intensity-modulated radiotherapy for head and neck cancer. International Journal of Radiation Oncology Biology Physics 70(3): 660-665.

6. Lin DS, Jen YM, Lee JC, Liu SC, Lin YS (2011) Recurrence of nasopharyngeal carcinoma in the parotid region after parotid-glandsparing radiotherapy. Journal of the Formosan Medical Association. 110(10): 655-660. 